\title{
LONG-TERM EFFECTS OF HAZARD ANTICIPATION TRAINING ON NOVICE DRIVERS MEASURED ON THE OPEN ROAD
}

\author{
Thalia Taylor ${ }^{1}$, Kathleen Masserang ${ }^{1}$, Anuj Pradhan ${ }^{2}$, Gautam Divekar ${ }^{1}$, Siby Samuel ${ }^{1}$, \\ Jeffrey Muttart $^{1}$, Alexander Pollatsek ${ }^{1}$, \& Donald Fisher ${ }^{1}$ \\ ${ }^{1}$ University of Massachusetts, Amherst, Massachusetts, USA \\ ${ }^{2}$ National Institute of Child Health and Human Development, Bethesda, Maryland, USA \\ Email: thaliaggtaylor@gmail.com
}

\begin{abstract}
Summary: (a) The purpose of this study was to determine whether novice drivers that were trained to anticipate hazards did so better than novice drivers who were not so trained immediately after training and up to one year after training occurred. (b) Novice drivers who had held their restricted license for about one month were randomly assigned to a PC-based hazard anticipation training program (RAPT) or a placebo (control) training program. The programs took about one hour to complete. The effects of training were assessed in a field drive by using patterns of eye movements to assess whether drivers anticipated a potential unseen hazard. (c) The effects of training persisted over time. In the field test immediately after training, the RAPT group anticipated the hazards $65.8 \%$ of the time whereas; the control group anticipated them only $47.3 \%$ of the time. Six or more months later, the groups were brought back for a second field test and the effects of training did not diminish; the RAPT group anticipated the hazards $61.9 \%$ of the time compared to $37.7 \%$ for the control group.
\end{abstract}

\section{OBJECTIVES}

Teen drivers are not a homogeneous group when it comes to the status of their licensure. In today’s environment where Graduated Driver Licensing (GDL) programs are now the norm, some teen drivers have a learner's permit, others a restricted license, and still others a full license. Among those with a restricted license, some have relatively little driving experience, others much more. Our research is primarily focused upon what we will define as newly-licensed drivers. These are teens between the ages of 16 and 18 years old who have been driving independently with an intermediate stage license for twelve months or less (recognizing, of course, that not all newly-licensed drivers are teens). It is these drivers who are most at risk. They are up to 13 times more likely to crash than learner's permit drivers per vehicle mile (Williams, 2003; Williams, Preusser, Ferguson, \& Ulmer, 1997) and up to five times more likely to crash during the first month of licensure than teen drivers who have had their license 12 months or more (Mayhew, Simpson, \& Pak, 2003; McCartt, Shabanova, \& Leaf, 2003).

There are at least three general areas in which novice drivers' behaviors differ radically from more experienced drivers. Specifically, these teens are less likely than experienced drivers to look at locations where hazards are likely to materialize (Pradhan, Hammel, DeRamus, Pollatsek, Noyce, \& Fisher, 2005), to pay sustained attention to the forward roadway while performing secondary in-vehicle tasks (Chan, Pradhan, Knodler, Pollatsek \& Fisher, 2010; Klauer, Dingus, Neale, Sudweeks, \& Ramsey, 2006; McEvoy, Stevenson, \& Woodward, 2007; Wikman, Nieminen, \& Summala, 1998), and to control the speed, acceleration and position of their vehicle 
in order to avoid hazards (Fisher, Laurie, Glaser, Connerney, Pollatsek, Duffy, \& Brock, 2002; Sagberg \& Bjørnskau, 2006). Based on analyses of police crash reports, these appear to be the very behaviors that are contributing to high crash rates among teens (McKnight \& McKnight, 2003). In particular, McKnight and McKnight estimate that the failure to search ahead, to the side, and to the rear taken together were implicated in $42.7 \%$ of the crashes; the failure to pay attention was the cause of $23.0 \%$ of the crashes; and the failure to adjust the vehicle's speed appropriately was responsible for $20.8 \%$ of the crashes. Note that in the last category, high speeds (speeds in excess of $112.6 \mathrm{~km} / \mathrm{hr}$ [70 miles/hr]) accounted for only $1.5 \%$ of the crashes. And note that the novice drivers were under involved in alcohol related crashes.

If inexperience is indeed the cause of many of the crashes during the first few months of driving, and most of the evidence suggests that such is the case (Treat, Tumbas, McDonald, Shinar, Hume, \& Mayer, 1979), then it makes sense to provide drivers with the training they need to overcome this lack of experience. Several versions of such a training program have now been developed and evaluated both on a driving simulator (Pollatsek, Narayanaan, Pradhan \& Fisher, 2006) and in the field (Pradhan, Pollatsek, Knodler \& Fisher, 2009). This training, RAPT (Risk Awareness and Perception Training), is focused on hazard anticipation. There are several different versions of the training program. In the version used in this study, participants see a sequence of slides taken from the perspective of a driver encountering a scenario which requires him or her to anticipate a hazard. The participant must use the cursor to click on areas of the roadway from which a threat is most likely to emerge (Figure 1). The participant would have had to use the cursor to click on the area near the end of the arrow pointing to the hidden sidewalk. After seeing the sequence of slides for one scenario, the driver is then given an explanation of the hidden hazard along with a top down display that helps the participant visualize the nature of the hidden hazard.
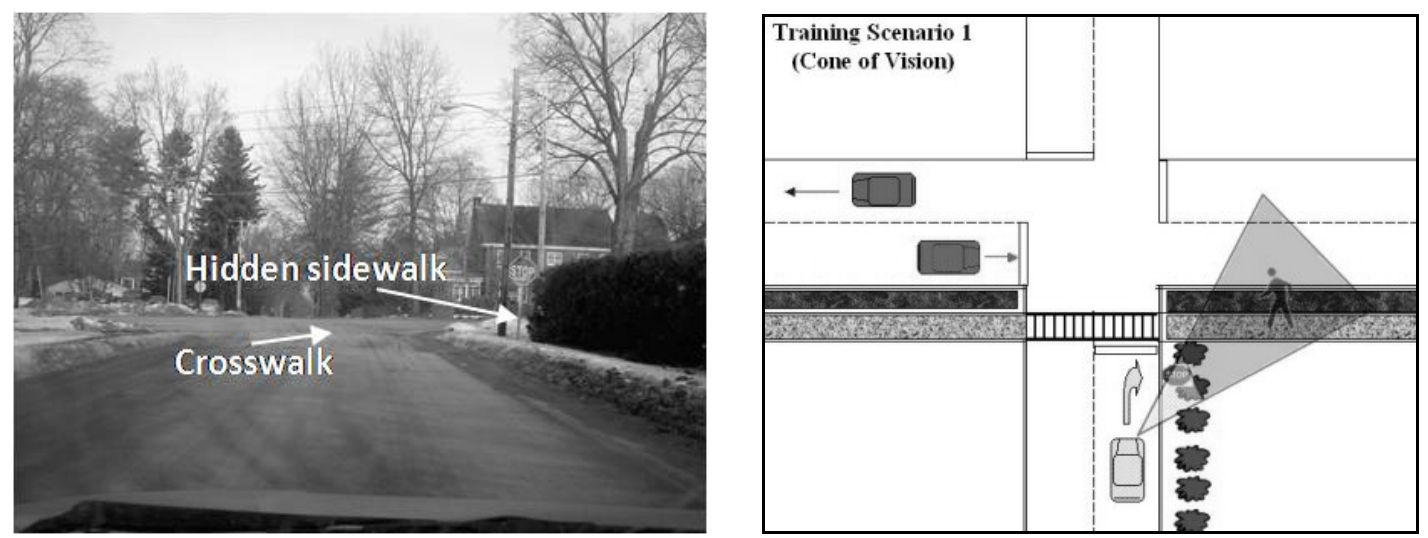

Figure 1. RAPT Training Program; View of the road ahead from driver's perspective (left) and screen used to explain hidden hazard (right)

Novice drivers trained with RAPT and evaluated immediately after training on a driving simulator were more likely to recognize hazards (52.1\%) than drivers who were given placebo training (28.1\%; placebo training is focused on the interpretation of various traffic control devices; Pollatsek et al., 2006). This advantage on a driving simulator extends up to a week after training (Pradhan, Fisher \& Pollatsek, 2006), where RAPT trained novice drivers now recognized $57.7 \%$ of the potential hazards whereas placebo trained drivers recognized only 
$35.4 \%$ of the potential hazards (the participants in this study were not the same ones used in the study of the effects of training immediately after exposure to the training program). Moreover, novice drivers trained with RAPT evaluated immediately after training on the open road were more likely to recognize hazards (67.4\%) than are drivers given placebo training (37.4\%).

The one remaining question, of critical practical importance, is whether these effects would be observed several months after training, extending throughout the period of time when the novice drivers are most at risk. There are good reasons to have believed that the program would have immediate training benefits. Not only did it provide novice drivers with knowledge about potential hazards which they did not have, but it also did so in an instructional environment in which they could and did make frequent errors. Error training has been shown to be highly effective in the near term (Ivancic \& Hesketh, 2000). More recently, error training has been shown to be effective in the intermediate term (three months), though with older drivers (Romoser \& Fisher, 2009). Still, it is an open question whether an hour's worth of training would still prove effective for a period of time extending over six months beyond the initial training for younger drivers. In fact, it is possible that little, if any, of the effect of the original training remains at such an interval. If a one hour training session leads to changes in hazard anticipation a long time after training has ended, this suggest that the reason for novice drivers' poor performance in hazard anticipation measures is that they lack the knowledge to anticipate hazards but will do so once trained, as outlined by McKnight \& McKnight in 2003.

\section{METHODS}

\section{Participants}

There were three participant groups. 28 participants were between the ages of 16 and 18, were licensed about a month before participation, and had taken a driver education course at a driving school in Greenfield, Massachusetts. Of these subjects, 15 received RAPT training, and 13 received a placebo-control training. The third group was made up of 15 participants between the ages of 26 to 55. All participants were paid.

\section{Design}

The key comparison was between the younger drivers who had been trained with the RAPT training program and those who had received placebo training consisting of reading a pamphlet on common road signs followed by a multiple choice quiz on the material. Immediately after the 40 minute training, these participants were fitted with an eye tracker before being tested on the first field drive. Then, 6-12 months after the first measurement session, participants returned for a second session wherein they were fitted with the eye tracker again before being tested on the same route as before. Originally, there were equal numbers of younger drivers randomly assigned to the two groups; however not all could be located for the delayed test. As the principal focus of the present study was to assess whether the effects of training were long-lasting, only the data for the 28 younger drivers who were tested on both sessions is reported in the main analyses. The experienced drivers served as another baseline for the training effect and we compared them to both the RAPT trained and the placebo-control group. This allowed us to assess whether the RAPT trained novice group had eye movements comparable to the 
experienced group; the experienced group was tested only once and received no training. All groups drove the same route during testing.

\section{Brief Description of Risk Awareness and Perception Training Program (RAPT)}

The RAPT program and the 11 types of scenarios on which the subjects were tested have been described in detail previously (Pradhan et al., 2009) and very briefly above. It is a simple program that can be run on any Microsoft Windows-operating PC. The training program displays sequences of photographs taken from the first person perspective of a driver maneuvering his way through downtown Amherst, MA. The scenarios spotlight an example of a hazard which is not immediately obvious. Of these hazardous scenarios, there were two general types. 1) Some situations contained a hidden threat which might emerge from behind an obscuring object. For example, a pedestrian might emerge from behind a truck stopped in the parking lane right before a midblock crosswalk. Or a bicyclist might emerge from behind a line of bushes hiding the sidewalk. 2) In other situations, a vehicle on the road ahead hidden by another vehicle could become a potential threat. For example, a vehicle in a left turn lane might suddenly pull into the adjacent lane in front of the driver. Each image in the sequence was presented for three seconds, during which participants had to click on all the locations in the photograph where they would look if they were actually driving through the scenario (example discussed above; see Figure 1). If the participant failed to click on a critical location in the sequence of images, a plan view of the scenario was shown that explained the potential threat and participants were given another chance to get it right. This could occur up to five times.

\section{Apparatus}

Eye tracker. A portable head-mounted eye-tracker (Mobile Eye developed by Applied Science Laboratories) collected eye-movement data during the drives. Once calibrated, eye movement data are converted to a crosshair, corresponding to the driver's point of gaze. These data were collected during field drives and later scored.

Participant vehicle. Each participant drove a four door sedan with a secondary breaking system that could be operated by a certified driving instructor.

\section{Field driving route}

Each participant drove the same 13 mile route in Greenfield, Massachusetts. It included rural, residential, and city driving scenarios. Throughout the drive, there were 11 scenarios for which the eye movement data were scored. The scenarios did not resemble any of the photographs in the RAPT program, so there were no cues to participants about the portions of the drive being measured. The 11 scenarios tested were in the same two general categories of hazard as in training: (a) a person or vehicle appearing from behind an obscuring object and (b) a vehicle ahead could become a potential threat. See Pradhan et al 2009 for a more detailed description of the 11 scenarios that the present ones were modeled on. Six of the scenarios were conceptually unlike the scenarios trained in RAPT but required use of the same general principles (far transfer). The other five scenarios were covered in the RAPT program but were not identical to the scenarios in the training session (near transfer). During measurement sessions, some of the 
scenarios (e.g. a truck blocking the beginning of a crosswalk) depended on the presence of external factors and thus were not always available for every participant; there were not always 11 observations available for each participant.

\section{Procedure}

The younger drivers were randomly assigned either to the RAPT or the placebo groups before the training session. They were then given either RAPT or placebo training on a computer before getting into the participant vehicle where they were fitted with a head mounted eye-tracker. Subjects were asked to drive normally. During the drive, the radio was not played and the only form of communication permitted consisted of spoken directions from the experimenter. Experienced drivers received the same treatment, except they did not receive any training. During all drives the participant drove, a certified driving instructor sat in the passenger seat, and an experimenter sat in the back seat.

After the drive, the data collected were calibrated before being scored. This generated a cross hair, corresponding to participant's point of gaze. If a participant's data could not be calibrated due to reflections from the sun or correctional eye wear, they were scored using gross pupil movements. There were up to two critical eye movements per scenario. All 11 scenarios were scored as follows: the driver was given a score of 1 if he or she recognized a potential threat and fixated the location(s) from which a threat could emerge. If the driver did not fixate any key locations a 0 was recorded, but if the driver fixated 1 of 2 key locations he or she received a 0.5 on the scenario. The scorer was blind to subject group.

We did not have tight control over the timing of the second test, as we could bring the participants back only when they agreed to come. The mean delay between tests was about eight months (255 and 236 days for the RAPT and placebo trained groups, respectively).

\section{RESULTS}

Results are summarized in Table 1. The RAPT training program improved glance behavior significantly over the glance behavior of the placebo control group. Averaged over the two sessions, there was a 21.4 percentage point difference between the groups, $t(26)=3.49, p=0.002$, $\mathrm{SE}=6.1 \%$. However, as the principal focus of the study was to examine whether the training effects were long-lasting, of primary interest was the difference between the two groups after an interval of 6 months or more after training. The size of the difference between the groups at the delayed test (session II) was actually slightly larger (24.2 percentage points), $t(26)=3.29$, $\mathrm{p}=0.002$, $\mathrm{SE}=7.3 \%$. The 18.5 percentage point difference between the two groups at session I was also significant, $\mathrm{t}(26)=2.50, \mathrm{p}=0.018, \mathrm{SE}=7.4 \%$. Both groups did slightly worse in session II than in session I; however, the $6.7 \%$ decline was not significant, $F(1,26)=2.82$, $\mathrm{p}>0.10$, $\mathrm{SE}=4.0 \%$. Also, unsurprisingly the interaction of the difference between the two groups and training session was not close to significant $(\mathrm{F}<1)$. However, the RAPT training did not put the drivers at the level of the experienced drivers. The experienced drivers performed significantly better than the average of the RAPT group's performance over the two sessions, $t(28)=2.72$, $\mathrm{p}=0.011, \mathrm{SE}=6.5 \%$. 
Table 1. Percentages of Correct Glance Behaviors Averaged over Scenarios

\begin{tabular}{ccccc}
\hline Group & $\begin{array}{c}\text { Average Session } \\
\text { I score (SD) }\end{array}$ & $\begin{array}{c}\text { Average Session II } \\
\text { score (SD) }\end{array}$ & $\begin{array}{c}\text { Average of Session I } \\
\text { \& II (SD) }\end{array}$ & $\begin{array}{c}\text { Difference between } \\
\text { Session I \& II (SD) }\end{array}$ \\
\hline RAPT Trained & $65.8(18.6)$ & $61.9(21.4)$ & $63.9(17.6)$ & $3.9(19.1)$ \\
Placebo Trained & $47.3(20.3)$ & $37.7(16.8)$ & $42.5(14.7)$ & $9.6(22.8)$ \\
Experienced & $81.6(18.0)$ & - & - & - \\
\hline
\end{tabular}

There are two other aspects of the data we should report. The first aspect is whether there were differences between the effects of training in the five near transfer scenarios and in the six far transfer scenarios. When broken down this way, there were many missing cells due to the fact that the scenario did not always materialize, as indicated in the methods section. (This was especially true of the near transfer scenarios.) As the overall difference between the groups was quite similar in test sessions I and II, the most powerful test was to average each participant's score over the two sessions. When this was done, there were clear differences between the RAPT-trained and placebo-trained groups for both near and far transfer. The difference for near transfer was 24.0 percentage points, $\mathrm{t}(23)=2.155, \mathrm{p}=.042$, $\mathrm{SE}=11.1 \%$, and for far transfer was 16.0 percentage points, $\mathrm{t}(26)=2.537, \mathrm{p}=.018, \mathrm{SE}=6.3 \%$. The second aspect is the mean in session I for the entire group tested on the first session. They were $67.3 \%$ for the RAPT-trained group and $51.0 \%$ for the placebo-trained group. Thus, the groups that are presented in Table 1 do not seem to be an obviously biased sample of the original randomly assigned groups.

\section{CONCLUSIONS}

The conclusions of our study are both straightforward and of significant interest. That is, somewhat contrary to our original expectations, there was absolutely no sign that the effects of training had decreased after a delay that was on the average of eight months. As indicated in the introduction, the period of the first 6-8 months on the road is a period of greatly increased crash rates, and furthermore, that failure to anticipate hazards of the type involved in the RAPT training program is likely to be a significant cause of this increased crash rate (McKnight and McKnight, 2003). As also indicated in the introduction, McKnight and McKnight outlined that it is unclear whether experienced novice drivers are clueless (they intend to drive well but lack the knowledge to do so) or careless (they may or may not have the knowledge but choose to drive poorly, regardless). These results support the conclusion that novice drivers mean to do well but are clueless until trained. As a result, our data indicate that the RAPT training program has the potential to decrease the accident rate through this critical period, making RAPT trained drivers more like the experienced drivers than the placebo trained drivers. Indeed, given that no decrease in the effect of the training was observed over this period, there is little reason to expect that the effects of the training will decrease until both groups become as experienced as the experienced drivers.

Needless to say, our data indicate that the training is not perfect and that the RAPT-trained drivers are still significantly worse at detecting hazards than the experienced drivers. However, there are limits on what can reasonably be accomplished in about an hour of training on an inexpensive training platform (i.e., a PC with no fancy peripheral devices). Although we will continue to strive to improve the RAPT training program, we believe that its present level of efficacy makes it a good candidate for a practical training program that can be implemented on a 
large scale and can significantly reduce crash rates among novice drivers, a rate which is some 13 times higher during the first month of driving than it is many years later.

\section{REFERENCES}

Chan, E., Pradhan, A. K., Knodler, M. A, Pollatsek, A. \& Fisher, D. L. (2010). Evaluation on a driving simulator of the effect on drivers' eye behaviors from distractions inside and outside the vehicle. Transportation Research F, 13, 343-353.

Ivancic, K. \& Hesketh, B. (2000). Learning from errors in a driving simulation: effects on driving skill and self-confidence. Ergonomics, 43, 1966 - 1984.

Klauer, S. G., Dingus, T. A., Neale, V. L., Sudweeks, J. D., \& Ramsey, D. J. (2006). The Impact of Driver Inattention On Near-Crash/Crash Risk: An Analysis Using the 100-Car Naturalistic Driving Study Data. Washington, D.C.: National Highway Traffic Safety Administration.

Mayhew, D. R., Simpson, H. M., \& Pak, A. (2003). Changes in collision rates among novice drivers during the first months of driving. Accident Analysis and Prevention,, 35, 683-691.

McCartt, A. T., Shabanova, V. I., \& Leaf, W. A. (2003). Driving experience, crashes and traffic citations of teenage beginning drivers. Accident Analysis and Prevention, 35, 311-320.

McKnight, J. A., \& McKnight, S. A. (2003). Young novice drivers: Careless or clueless. Accident Analysis and Prevention, 35, 921-925.

McEvoy, S. P., Stevenson, M. R., \& Woodward, M. (2007). The prevalence of, and factors associated with, serious crashes involving a distracting activity. Accident Analysis and Prevention 39, 475-482.

Pradhan, A. K., Fisher, D. L. \& Pollatsek, A. (2006). Risk Perception Training for Novice Drivers: Evaluating Duration of Effects on a Driving Simulator. Transportation Research Record, 1969, 58-64.

Pollatsek, A., Narayanaan, V., Pradhan, A., \& Fisher, D. L. (2006). The Use of Eye Movements to Evaluate the Effect of PC-Based Risk Awareness Training on an Advanced Driving Simulator. Human Factors, 48, 447-464

Pradhan, A. K., Hammel, K. R., DeRamus, R., Pollatsek, A., Noyce, D. A., \& Fisher, D. L. (2005). The Use of Eye Movements to Evaluate the Effects of Driver Age on Risk Perception in an Advanced Driving Simulator. Human Factors, 47, 840-852.

Pradhan, A. K., Pollatsek, A., Knodler, M. \& Fisher, D. L. (2009). Can younger drivers be trained to scan for information that will reduce their risk in roadway traffic scenarios that are hard to identify as hazardous? Ergonomics, 62, 657-673.

Romoser, M. \& Fisher, D. L. (2009) The effect of active versus passive training strategies on improving older drivers' scanning for hazards while negotiating intersections. Human Factors, 51, 652-668.

Sagberg, F., \& Bjørnskau, T. (2006). Accident Analysis \& Prevention. Hazard perception and driving experience among novice drivers., 3, 407-414.

Treat, J. R., Tumbas, N. S., McDonald, S. T., Shinar, D., Hume, R. D., \& Mayer, R. E. (1979). Tri-level study of the causes of traffic accidents: Final report Vol. I. Causal factor tabulation 
and assessments. Bloomington, IN: Indiana University, Institute for Research in Public Safety.

Wikman, A., Nieminen, T., \& Summala, H. (1998). Driving experience and time-sharing during in-car tasks on roads of different width. Ergonomics, 41(3), 358-372.

Williams, A. F. (2003). Teenage drivers: patterns of risk. Journal of Safety Research, 34(3), 515.

Williams, A. F., Preusser, D. F., Ferguson, S. A., \& Ulmer, R. G. (1997). Analysis of the fatal crash involvements of 15-year-old drivers. Journal of Safety Research, 28(1), 49-54. 